\title{
Acoustic probing of elastic behavior and damage in weakly cemented granular media
}

\author{
V. Langlois ${ }^{1, *}$ and X. Jia ${ }^{1,2, \dagger}$ \\ ${ }^{1}$ LPMDI, Université Paris-Est, 5 Boulevard Descartes, 77454 Marne-la-Vallée Cedex 2, France \\ ${ }^{2}$ Institut Langevin, ESPCI ParisTech, CNRS UMR No. 7587, 1 Rue Jussieu, 75005 Paris, France
}

(Received 17 September 2013; published 19 February 2014)

\begin{abstract}
We investigate the elastic behavior and damage of weakly cemented granular media under external load with ultrasound. The cementation controlled experiments are performed by freezing the capillary liquid at the bead contact in a dense glass or polymeric [poly(methyl methacrylate)] bead pack wet by tetradecane of volume fraction $\phi=0.1 \%-4 \%$. When the pendular rings are solidified, an abrupt increase by a factor of 2 in the compressional wave velocity is observed. We interpret the data in terms of effective medium models in which the contact stiffnesses are derived by either a bonded contact model [P. J. Digby, J. Appl. Mech. 48, 803 (1981)] or a cemented contact model [J. Dvorkin, A. Nur, and H. Yin, Mech. Mater. 18, 351 (1994)]. The former fails to quantitatively account for the results with a soft cement relative to the grain, whereas the latter considering the mechanical properties of the cement does apply. Moreover, we monitor the irreversible behavior of the cemented granular packs under moderate uniaxial loading $(<1.3 \mathrm{MPa})$ with the correlation method of ultrasound scattering. The damage of the cemented materials is accompanied by a compressional wave velocity decrease up to $60 \%$, likely due to the fractures induced at the grain-cement interfaces.
\end{abstract}

DOI: 10.1103/PhysRevE.89.023206

PACS number(s): 43.35.+d, 81.05.Rm, 46.40.-f

\section{INTRODUCTION}

Adding a small amount of liquid has a drastic effect on the mechanical properties of granular materials such as angle of avalanche and failure [1-4]. The tiny volume of liquid makes adhesive forces between grains and changes a granular medium from one with only repulsive interactions between grains to one with both repulsive and attractive interactions via liquid capillary bridges. In addition to the cohesion on static assemblies, the wetting liquid also affects the granular dynamics by the lubrication of solid friction and viscous dissipation $[5,6]$. As shown in weakly wet granular media, the highly increased acoustic absorption by a factor of 5 is due to the viscous loss in the liquid films adsorbed at the grain surface, strongly sheared at the contact area during the wave propagation [7]. However, for compressed granular packs, these capillary liquids only modify slightly the elastic stiffness of the materials $(\sim 10 \%)$ basically determined by the confining pressure [8].

When the wetting liquid is solidified, e.g., by freezing the contact between grains, which causes them to become bonded or cemented, it causes a very important increase in the contact stiffness and accordingly in the effective elastic modulus of the granular materials [9-14]. To some extent, weakly cemented granular materials can be compared to another class of cohesive granular media, i.e., fine powders, in which the cohesion stems from surface forces acting at the contact zone and the shear strength depends on the interplay between adhesion and friction [15]. Nevertheless, fine powders generally have high porosity and undergo important plastic compaction under compression.

Cemented dense granular materials are involved in numerous fields including the pharmaceutical industry, civil engineering, and geology [10-14]. Understanding the elastic

\footnotetext{
*langlois@univ-mlv.fr

${ }^{\dagger}$ xiaoping.jia@espci.fr
}

and plastic behavior of these porous granular materials can provide key insights into phenomena such as landslides and formations of frozen soil. For small cement concentration [Fig. 1(a)] in the pendular regime [4], Dvorkin et al. developed a cemented contact theory to estimate the effective elastic moduli of a dense random pack of identical elastic spheres with an elastic binder, i.e., cement [11]. Two types of cemented contacts are considered: without [Fig. 1(b)] or with prestress [Fig. 1(c)]. This model qualitatively agreed with acoustic velocities measured in various cemented granular packs: glass beads and epoxy, sand and ice, or sintered glass beads. However, the quantitative comparison between theory and experiment was still lacking, particularly for small cement content, due to the inhomogeneous distribution of cement around the particles [11]. Another issue still remains unclear: Is the cemented contact stiffness pressure sensitive? If so, is the process elastic or plastic at the contact level $[12,13]$ ?

In this work we address these issues by acoustically monitoring the weakly cemented glass and polymeric bead packs under external load. The relationship between the acoustic velocity and cement content is explored by evenly wetting the grains with small amounts of tetradecane (volume fraction $\phi<4 \%$ ), solidified at a temperature below its freezing point. These data will be analyzed with the effective medium theory (EMT) based on a bonded contact model [9] and a cemented contact model [11], respectively. We investigate the pressure dependence of the acoustic velocity in the cemented granular packs and monitor the material damage under uniaxial loading and unloading with the correlation technique of ultrasound scattering, sensitive to the structural change of the materials down to scales of the grain contacts $[16,17]$.

\section{EXPERIMENT}

\section{A. Preparation of cemented granular materials}

Our granular media are composed of slightly polydisperse glass and polymeric [poly(methyl methacrylate) (PMMA)] 


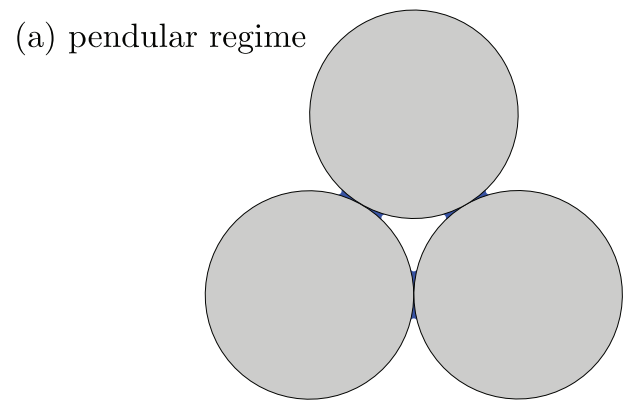

(b) without prestress (c) with prestress $P_{0}$

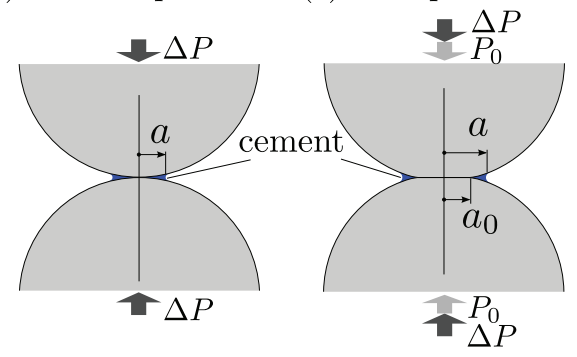

FIG. 1. (Color online) (a) Schematic illustration of a small amount of cement distributed in a granular pack. Cemented contacts are prepared (b) without or (c) with prestress $P_{0}$. Here $\Delta P$ corresponds to the external stress applied to the cemented contacts.

beads of diameter $d=0.7 \pm 0.1 \mathrm{~mm}$. To control the distribution of cementation inside granular samples, we evenly wet the grains with small amounts of tetradecane by stirring the mixture over 1 min [3,7], confirmed by optical microscopic observation. The tetradecane volume fraction $\phi$ relative to the bead volume (or cement content) varies from $0.1 \%$ to $4 \%$. The wet media are then filled into an oedometric cell $30 \mathrm{~mm}$ in diameter and $12 \mathrm{~mm}$ high. An axial stress of $600 \mathrm{kPa}$ [Fig. 2(a)] is applied twice to preload the samples; the initial bead packing density is $\Phi=0.62 \pm 0.01$ for glass bead packs and $0.59 \pm 0.01$ for PMMA bead packs, respectively. These packing densities are smaller compared to the value of random dense packing of about 0.64 , likely due to the capillary force between the grains. The characteristics of the solid particles and tetradecane are given in Table I.

The cemented granular media are obtained by putting the oedometric cell containing the wet granular media inside a refrigerator and lowering the temperature below the freezing point of the wetting liquid. An isolator is placed around the cell to ensure a more homogeneous thermal distribution. A broadband short pulse $(\sim 2 \mu \mathrm{s})$ is generated by a large longitudinal transducer (E) $30 \mathrm{~mm}$ in diameter centered at $500 \mathrm{kHz}$. The ultrasound transmission is received either by the same large transducer $(\mathrm{R})$ to adequately detect the low-frequency coherent compressional wave $(\sim 100 \mathrm{kHz})$

TABLE I. Characteristics of beads and tetradecane.

\begin{tabular}{lccc}
\hline \hline Material & $\rho_{g}\left(\mathrm{~kg} / \mathrm{m}^{3}\right)$ & $G_{g}(\mathrm{GPa})$ & $\nu_{g}$ \\
\hline glass & 2450 & 26.2 & 0.28 \\
PMMA & 1190 & 2.43 & 0.31 \\
tetradecane (solid) & 800 & 1.10 & 0.34 \\
\hline \hline
\end{tabular}

(a)
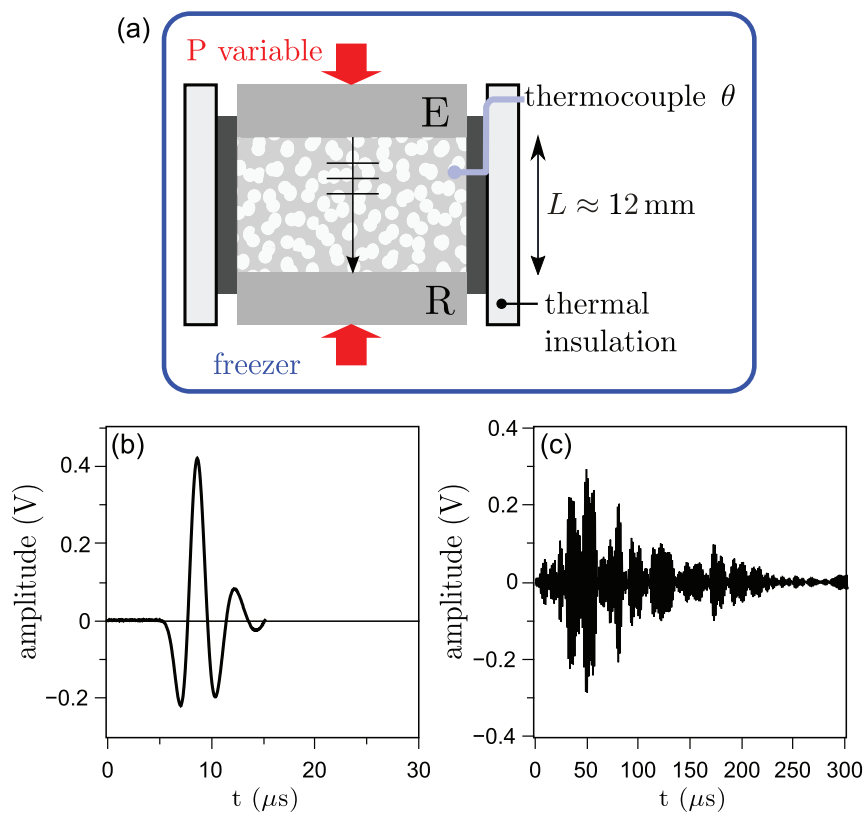

FIG. 2. (Color online) (a) Experimental setup. (b) Typical pulse of a coherent compressional wave. (c) Codalike scattered waves obtained with a high-pass filter.

[Fig. 2(b)] or by a small detector $2 \mathrm{~mm}$ in diameter (not shown) to probe the incoherent acoustic speckles [Fig. 2(c)]. These codalike acoustic speckles are obtained by a narrow-band filter of $550-850 \mathrm{kHz}$; these waves correspond to the scattered waves through the heterogeneous medium, which are configuration specific to the contact network and thus sensitive to any structural change [18].

Two methods are employed in this work to measure the time-of-flight velocity of the coherent compressional wave $V_{P}$. One determines the absolute value of $V_{P}$ via the Hilbert transformation [19] with a reference signal obtained from the transducers $\mathrm{E}$ and $\mathrm{R}$ in contact; this method is used below (Sec. II B) to make a quantitative comparison of our data with the EMT prediction. The other method for determining $V_{P}$ measures the time of flight from the first extreme peak [Fig. 2(b)]; this is suitable for a quick evaluation of the relative change in $V_{P}$ under loading and unloading (Sec. IIC).

\section{B. Acoustic velocity versus cement content and prestress effect}

Figure 3(a) shows a typical evolution of the acoustic velocity $V_{P}$ of compressional wave during the transition from a wet bead pack at $\phi=0.82 \%$ under stress to a cemented granular material when lowering the temperature (first cooling under high prestress). An abrupt increase, by nearly a factor of 2 , is observed in the acoustic velocity $V_{P}$ when approaching the freezing point of tetradecane around $\theta_{\varphi}=6^{\circ} \mathrm{C}$. In order to examine the prestress effect on the cemented material (Fig. 1), we vary the uniaxial stress $P_{0}$ applied to the wet bead pack; the cemented sample with a low prestress (second cooling) is obtained after the coolingheating cycle at high prestress to avoid possible experimental parasites. These cemented samples will be submitted to further loading-unloading experiments. 

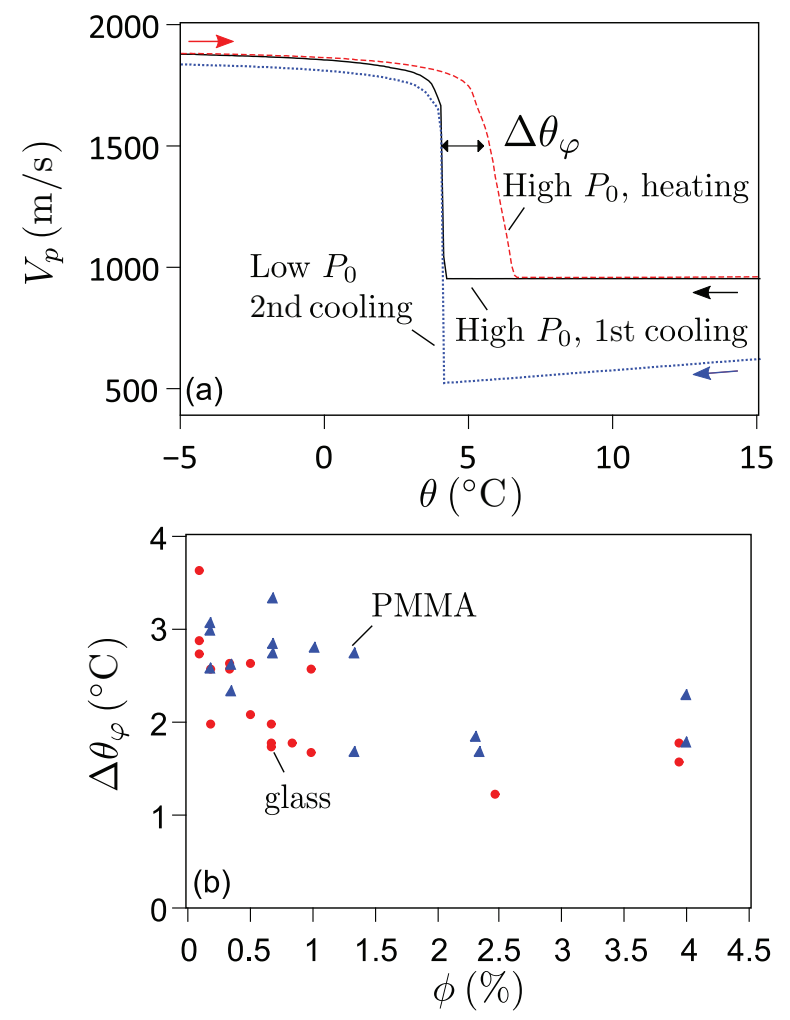

FIG. 3. (Color online) (a) Acoustic velocity $V_{P}$ of the compressional wave measured in wet and cemented glass bead packs as a function of temperature and prestress $P_{0}$. (b) Difference of the phase transition temperature between heating and cooling processes versus the cement content.

It is worth noting that the temperature $\theta_{\varphi}$ of the phase transition at which the abrupt change of $V_{P}$ occurs when cooling the wet materials is systematically lower than that when heating the cemented materials by $\Delta \theta_{\varphi}$. Figure 3(b) illustrates the evolution of $\Delta \theta_{\varphi}$ versus the volume fraction of the wetting liquid $\phi$. The results likely imply a supercooled state of tetradecane when lowering $\theta$ below $\theta_{\varphi}$ : The smaller the amount of liquid, the more pronounced the phenomenon is.

Figures 4(a) and 4(b) depict the acoustic velocities $V_{P}$ measured as a function of cement or wetting liquid content in the above cemented glass and PMMA bead packs at $\theta=-5^{\circ} \mathrm{C}$, respectively. The cemented samples are prepared with distinct prestresses at low $P_{1}=40 \pm 20 \mathrm{kPa}$ and high $P_{2}=1240 \pm$ $20 \mathrm{kPa}$ to simulate the two geometries of cemented contact (Fig. 1). The acoustic velocities measured at the wet granular packs at $\theta=15^{\circ} \mathrm{C}$ are also presented in the insets of Fig. 4. The latter are much smaller than those in the cemented media and follow roughly the scaling law $V_{2} / V_{1}(\sim 1.8) \propto\left(P_{2} / P_{1}\right)^{1 / 6}$, predicted by the Hertzian contact $[5,18,20]$. The acoustic velocity in the cemented medium significantly increases with the amount of cement $\phi$, particularly at small value [11], while $V_{P}$ of the wet media is insensitive to the liquid content, as mentioned before.

The effect of the prestress appears more important in the cemented PMMA beads than in cemented glass beads. Figure 4(c) shows the relative change of $V_{P}$ measured for two distinct prestresses as a function of cement content. Increasing
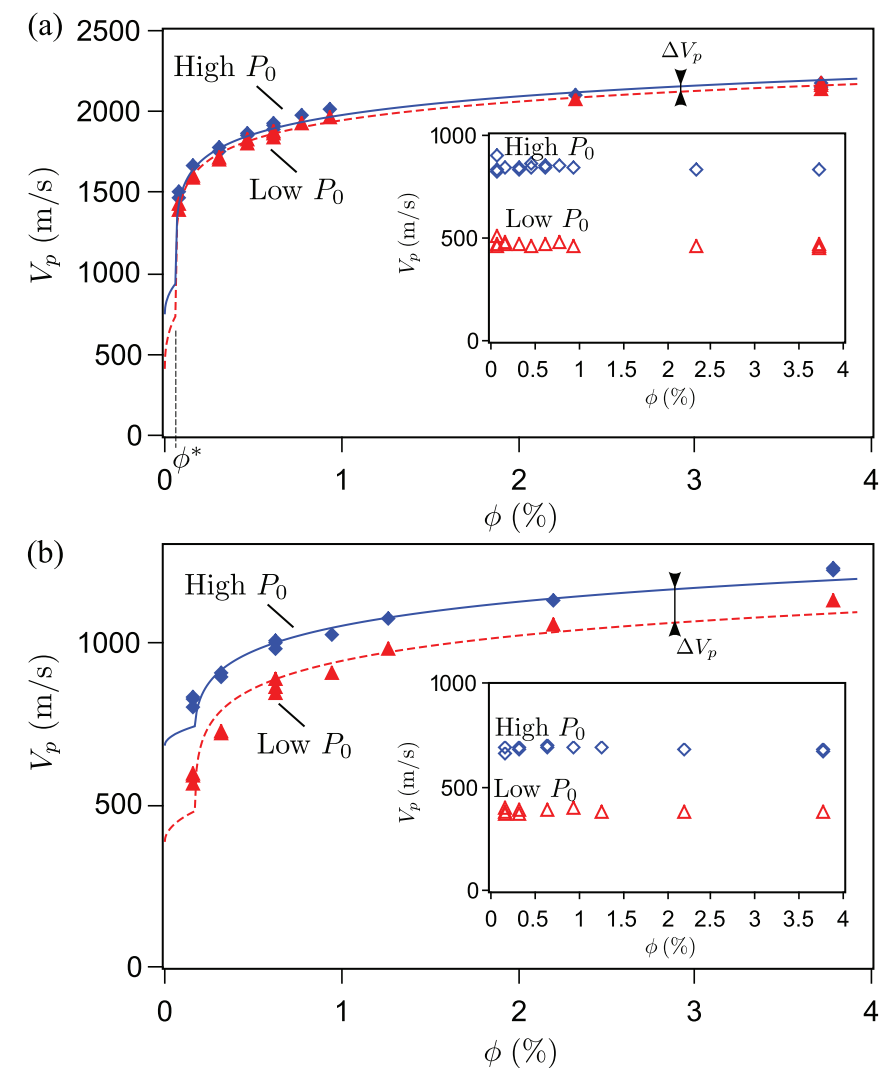

(c)

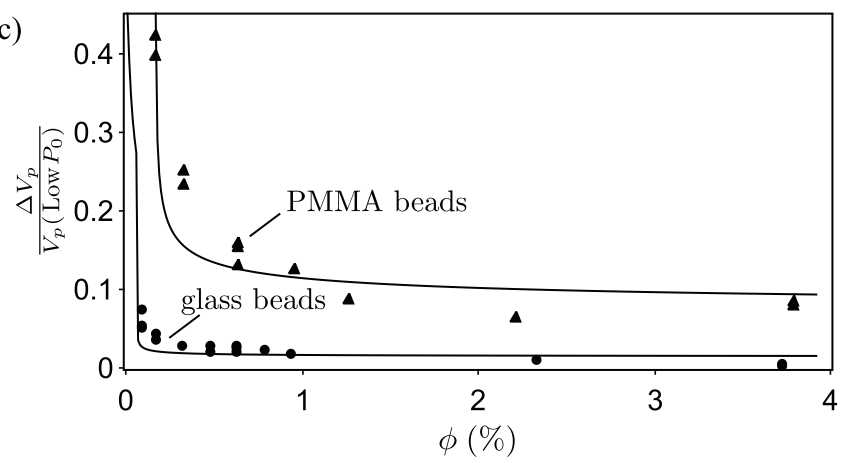

FIG. 4. (Color online) Acoustic velocities $V_{P}$ measured as a function of the volume fraction of tetradecane in (a) cemented glass and (b) PMMA packs, prepared with prestress $P_{0}=40 \mathrm{kPa}$ (triangle) and $1240 \mathrm{kPa}$ (diamonds). (c) Relative increase in acoustic velocity of cemented granular packs at $\theta=-5^{\circ} \mathrm{C}$ due to the prestress $P_{0}$. The solid and dashed lines correspond to the predictions by the cemented contact model for high and low prestresses, respectively.

$\phi$ decreases the effect of prestress on $V_{P}$. Moreover, we observe that there is a considerable difference of $V_{P}$ between the cemented and wet media for glass beads compared to that for the PMMA beads [Figs. 4(a) and 4(b)]. This implies that the elastic modulus of very weakly cemented granular media may depend on the interplay of the bulk properties between grains and cements.

\section{Monitoring of the cemented material damage under uniaxial load}

Acoustic methods including diffusing wave spectroscopy [16,17] and nonlinear acoustics [21] provide very 

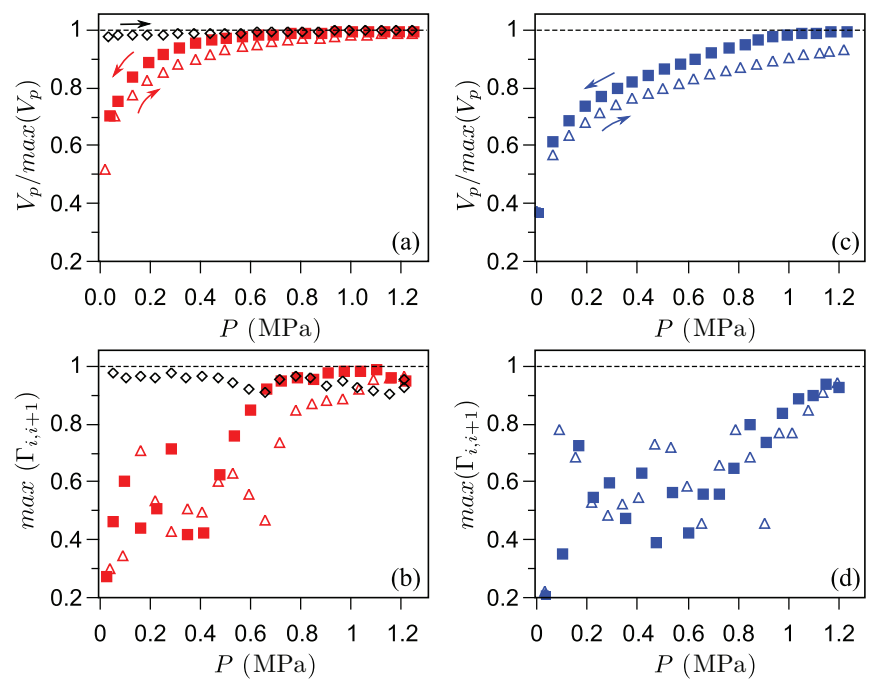

FIG. 5. (Color online) (a) Evolutions of the normalized acoustic velocity $V_{P}$ and (b) the resemblance of codalike scattered waves during loading (diamonds), unloading (squares), and reloading (triangles) in a cemented glass bead pack prepared at low prestress. Similar measurements were performed during (c) unloading and (d) reloading in the cemented sample prepared at high prestress. The cement content is $\phi \approx 0.65 \%$.

useful nondestructive evaluation of the damage in heterogeneous materials (e.g., cracks). For noncohesive granular packs, the high-amplitude acoustic wave allows one to explore the Hertzian nonlinearity between the grains via solitonlike propagation or harmonic generation [22]; it can also cause irreversible sound-matter interaction such as the contact network rearrangement $[23,24]$. Here we probe nondestructively the change of cemented granular materials under axial compression, combining the velocity measurement of the coherent wave and the correlation technique of scattered waves in the linear acoustic regime. Figure 5(a) presents the typical evolutions of the acoustic velocity in a cemented glass bead pack with $\phi=0.65 \%$, prepared under a low prestress of $P_{1}$ $\approx 40 \mathrm{kPa}$, during a cycle of loading, unloading, and reloading up to $1200 \mathrm{kPa}$. A slight increase of the acoustic velocity $V_{P}$ by $3 \%$ is observed during first loading [Fig. 6(a)], where the different data (symbols) correspond to the cement content ranging from $0.2 \%$ to $3.7 \%$. Upon unloading, the velocity data show certain reversible behavior at the early stage by a small decrease, followed by an important irreversible decrease. Such a decrease of the acoustic velocity is also observed in noncohesive granular packs where the contact stiffnesses arise from the external load. Here the unloading could induce a progressive debonding of the contact. The nonzero value of the acoustic velocity, when the external load is removed, indicates, however, that the grains remain partially bonded [9]. When reloading, $V_{P}$ increases again, however, presenting certain hysteretic behavior.

To further investigate this irreversible behavior on scales of the contact, we present in Fig. 5(b) the variations of the configuration-specific scattered waves during the above external loading $\Delta P$. The degree of resemblance $\Gamma_{i, j}$ between two coda signals $S_{i}(t)$ and $S_{j}(t)$ recorded at two successive
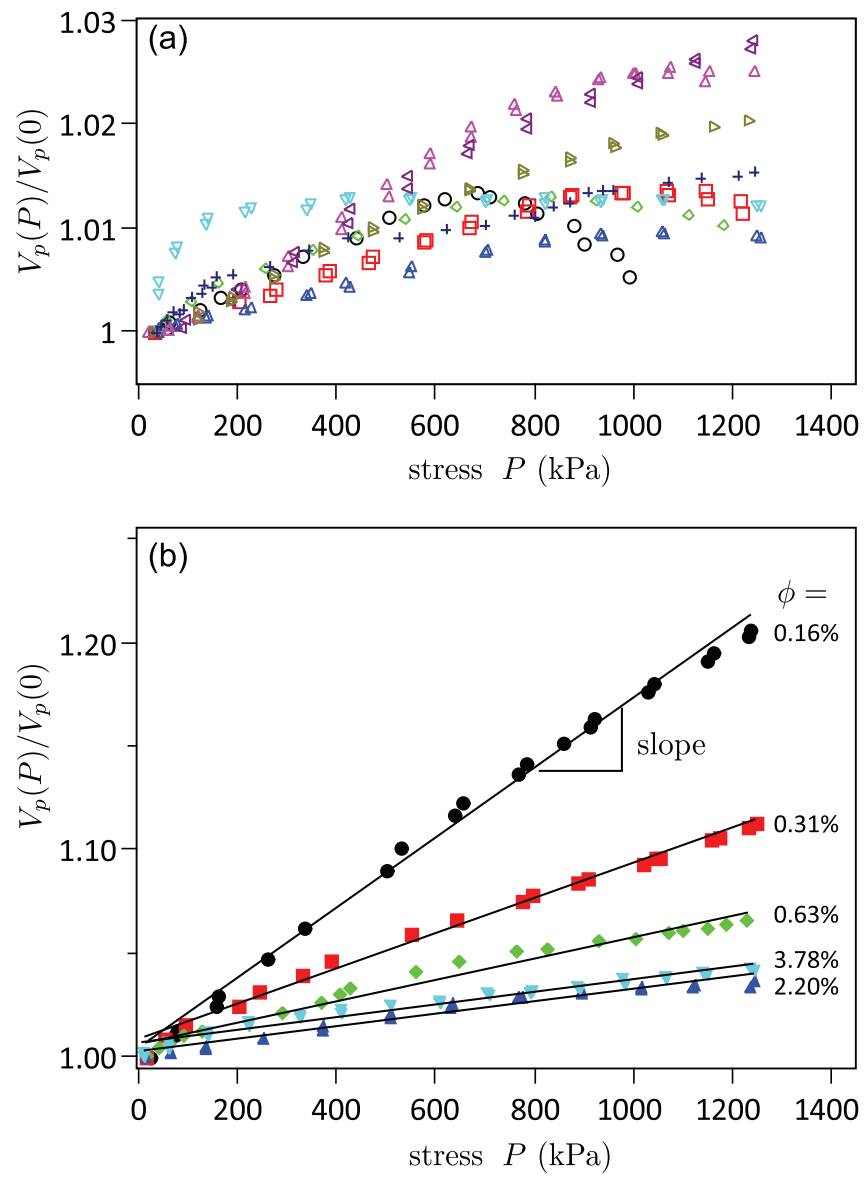

FIG. 6. (Color online) Evolutions of the acoustic velocity $V_{P}$ during the first application of loading on (a) cemented glass and (b) PMMA bead packs versus different cement content $\phi$.

loading steps $(i, j)$ is given by

$$
\Gamma_{i, j}(\tau)=C_{i, j}(\tau) /\left[C_{i, i}(0) C_{j, j}(0)\right]^{1 / 2},
$$

where $C_{i, j}(\tau)=\int S_{i}(t) S_{j}(t-\tau) d t$ is the cross-correlation function of $S_{i}(t)$ and $S_{j}(t)$ and $\tau$ the lag time. The maximum value of $\Gamma_{i, j}, \max \left(\Gamma_{i, j}\right)$, characterizes their resemblance and therefore probes the modification of the cemented contact networks under incremental change in $\Delta P$, along which the codalike scattered waves propagate [17]. Figure 5(b) reveals that the cemented network almost remains unchanged with a constant $\max \left(\Gamma_{i, j}\right) \approx 0.9$ during the first loading $(<1.3 \mathrm{MPa})$ and the early stage of unloading. For further unloading $(<0.5$ MPa) $\Gamma_{i, j}$ starts to vary significantly and is accompanied by a velocity decrease larger than $3 \%$; this would be caused by the development of important damage inside the cemented contact network. Under reloading, the fractured medium exhibits the rearrangement as in the noncohesive granular pack.

Such irreversible behavior is also observed in cemented glass bead packs prepared at high prestress $P_{2} \approx 1240 \mathrm{kPa}$ [Fig. 5(c)]. Under unloading, the sound velocity exhibits a large velocity decrease at a much earlier stage than the above sample cemented at low pressure. In the meantime, the resemblance of the coda waves [Fig. 5(d)] shows the important variation that would indicate a large fluctuating damage developed in the cemented contact network when the 
prestress is removed. Note, nevertheless, that the heights of these two cemented samples almost remain unchanged during the loading.

The pressure dependence of the acoustic velocity observed during the first loading [Fig. 6(a)] is even more pronounced with cemented PMMA bead packs prepared under low prestress $P_{1}$; the velocity increase $\Delta V_{P} / V_{P}$ reaches $20 \%$ [Fig. 6(b)]. For cemented glass bead packs the pressure dependence of $V_{P}$ is insensitive to the cement content $\phi$, whereas for cemented PMMA bead packs $V_{P}$ does depend on $\phi$, according to a nearly linear relationship. Moreover, the smaller $\phi$ is, the stronger the pressure sensitivity is. This result again indicates that the mechanical responses of cemented granular packs depend on both the interfacial properties and those of the grain materials.

\section{COMPARISON WITH EFFECTIVE MEDIUM MODELS}

In this section we compare the measured acoustic velocity $V_{P}$ with EMTs, based on two different models for calculating the contact forces between bonded elastic spheres. To do so, we first recall in Sec. III A the EMT relating the elastic wave velocities to the contact stiffnesses and describe a few purely interfacial models. Then, in Sec. III B, we establish the relationship between the size of the cemented contact and the cement content $\phi$. This allows the calculation of $V_{P}$ as a function of $\phi$ by the interfacial model in Sec. III B and the cemented contact model in Sec. III C, respectively. We finally discuss in Sec. III D how the cement content and particularly its bulk property affect the elastic behavior of weakly cemented granular materials.

\section{A. Effective medium theory based on interfacial bonding models}

Within the framework of the EMT, the incremental elastic bulk $K$ and shear $G$ moduli of a random packing of identical spheres of radius $R$ can be related to the normal and tangential contact stiffnesses $D_{n}$ and $D_{t}$ by [9]

$$
\begin{aligned}
& K=(Z \Phi / 12 \pi R) D_{n}, \\
& G=(Z \Phi / 20 \pi R)\left(D_{n}+3 D_{t} / 2\right),
\end{aligned}
$$

where $Z$ is the mean coordination number and $\Phi$ the packing density of spheres. Using the relationships $V_{P}^{2}=$ $(K+4 G / 3) / \rho$ and $V_{S}^{2}=G / \rho$ for an isotropic elastic medium where $\rho=\Phi \rho_{g}$ and $\rho_{g}$ is the material density of spheres, the compressional and shear wave velocities $V_{P}$ and $V_{S}$ may be written as [10]

$$
\begin{aligned}
& V_{P}^{2}=\left(3 Z / 20 \pi R \rho_{g}\right)\left(D_{n}+2 D_{t} / 3\right), \\
& V_{S}^{2}=\left(Z / 20 \pi R \rho_{g}\right)\left(D_{n}+3 D_{t} / 2\right) .
\end{aligned}
$$

In such mean-field theory, one assumes that the spatial fluctuation of contact density and stiffness can be ignored and the affine approximation is valid with $Z$ being assumed constant. All these assumptions appear to hold for the long-wavelength small-amplitude sound propagation in jammed cohesive granular material. Indeed, the variations of $\mathrm{Z}$ due to either the network rearrangements by nonlinear acoustic waves [24] or the contact recruitment by the applied compression $P$ [25] could be neglected in the cemented packs investigated in this work.

Several models were developed to compute the contact stiffness between two elastic spheres [26,27]. For a nonadhesive contact compressed by a normal force $F_{n}, D_{n}=$ $4 G_{g} a /\left(1-v_{g}\right)$ and $D_{t}=8 G_{g} a /\left(2-v_{g}\right)$ are predicted by the standard Hertz-Mindlin theory, where $G_{g}$ and $v_{g}$ are the shear modulus and Poisson coefficient of spheres and $a \propto F_{n}^{1 / 3}$ is the contact radius. The tangential stiffness $D_{t}$ stems from the nonslip contact area of radius $\sim a$ when the tangential force $F_{t}$ is well below $\mu F_{n}$, the sliding threshold between the smooth spheres ( $\mu$ is the friction coefficient) [26]. Increasing tangential force reduces $D_{t}$ due to the growth of a microslip annulus initiated at the edge of the contact area. For an adhesive contact, the above scaling holds for $D_{n}$ and $D_{t}$ where the contact radius $a$ depends on both the normal force and adhesion (e.g., van der Waals forces) as given by the Johnson-Kendall-Roberts model [15,26]. Digby proposed a phenomenological model for two elastic spheres initially bonded (nonslip condition) over a finite area of radius $b \leq$ $a$ (equality applies when the applied load is absent). This model, referred to here as the bonded contact model, gives two independent normal and tangential stiffnesses [9]

$$
\begin{aligned}
D_{n} & =4 G_{g} a /\left(1-v_{g}\right), \\
D_{t} & =8 G_{g} b /\left(2-v_{g}\right)
\end{aligned}
$$

and describes well the results obtained in sintered granular materials [28].

Note that all the above models take into account only the material properties of grains, the external load, and the interfacial forces (frictional or adhesive); however, the bulk properties of the binder or cement are ignored (see Sec. III C).

\section{B. Cement distribution versus wetting phenomena}

To compare the prediction by the bonded contact model with sound velocities $V_{P}$ measured as a function of the cement content $\phi$, one needs to establish the relationship between the radius $a$ of the cemented or bonded contact and $\phi$. Preceding the cementation by freezing, the wetting process in granular packs has made significant progress the past decade [3-5]. Instead of the formation of a capillary bridge at the bead contact, i.e., deposition 1 [Fig. 7(a)], the wetting liquid is first distributed as thin layers on a rough surface between two spheres with a maximum volume fraction $\phi^{*}$, due to the trapping by asperities (deposition 2). As shown by Mason et al. [3], beyond the threshold volume fraction $\phi>\phi^{*}$, the excess of the added liquid migrates towards the contact point between spheres, forming the hourglass-shaped meniscus (deposition 3).

When a prestress is applied, most of the wetting liquid at the contact area is squeezed out [Fig. 1(b)]. The relationship between $\phi$ and $a$ or $\alpha(=a / R)$ may be expressed for deposition 3 as

$$
\begin{gathered}
\alpha=\left[\alpha_{0}^{2}+2 \phi / 3+\left(4 \alpha_{0}^{2} \phi / 3\right)^{1 / 2}\right]^{1 / 2} \text { for } \phi<\phi^{*}, \\
\alpha=\left\{2 \phi^{*} / 3+\alpha_{0}^{2}+\left[4 \alpha_{0}^{2} \phi^{*} / 3+16\left(\phi-\phi^{*}\right) / 3 Z\right]^{1 / 2}\right\}^{1 / 2} \\
\text { for } \phi<\phi^{*}
\end{gathered}
$$


(a) Cement deposition

Deposition $1 \quad$ Deposition 2 Deposition $3\left(\phi>\phi^{*}\right)$
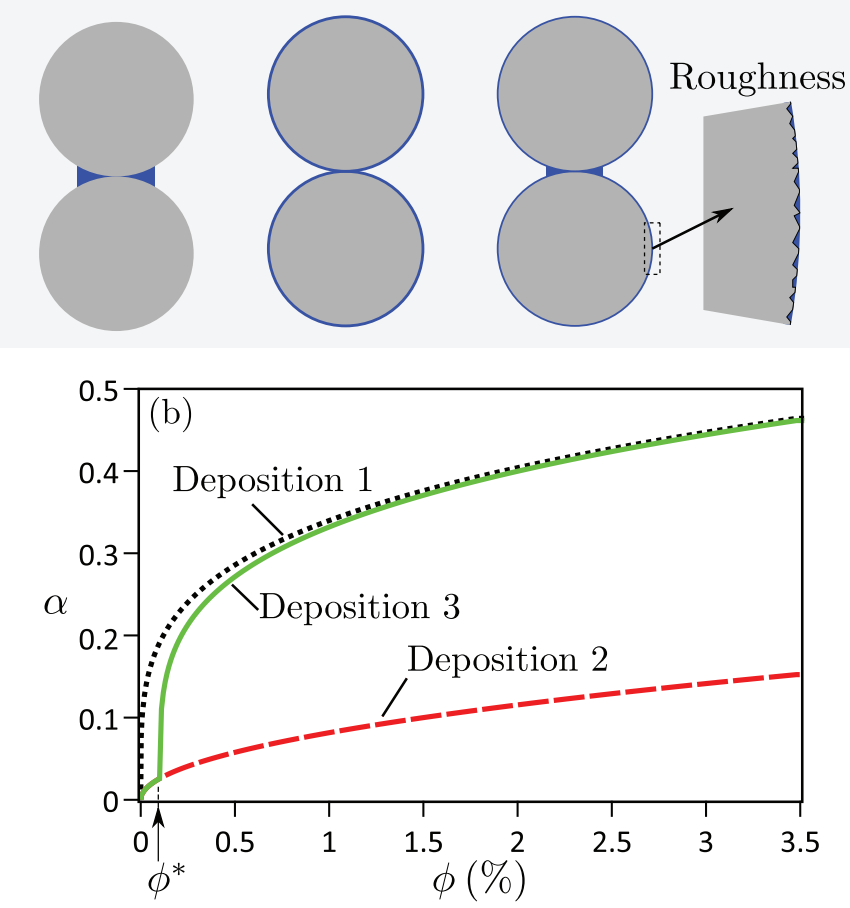

FIG. 7. (Color online) (a) Different depositions of the wetting liquid or cement. (b) Variations of the cemented contact radius $\alpha$ as a function of the cement content $\phi$ at different depositions.

where $\alpha_{0}=a_{0} / R$ is the normalized radius of the prestressed contact area. The detailed derivation of Eq. (5) is given in Appendix. If one neglects the filling of liquid on the surfaces of rough spheres $\phi^{*}=0$ and the prestress effect $\alpha_{0}=0$, Eq. (3b) reduces to $\alpha=(16 \phi / 3 Z)^{1 / 4}$, corresponding to deposition 1 , and Eq. (5a) leads to $\alpha=(2 \phi / 3)^{1 / 2}$, recovering deposition 2 , the two depositions of cement previously considered [12]. Figure 7(b) illustrates the variation of the normalized radius $\alpha$ as a function of $\phi$ for different arrangements. As mentioned above, deposition 2 is not relevant in this work due to the wetting phenomena.

Combining Eqs. (3)-(5), we plot in Fig. 8 the computed acoustic velocities $V_{P}$ with the Digby model for bonded glass and PMMA bead packs prestressed at low $P(=40 \mathrm{kPa})$, as a function of the cement content $\phi$. More specifically, by fitting $V_{P}$ measured at $\theta=15^{\circ} \mathrm{C}$ in the wet granular packs under $P$, we first extract the product $Z a$ from Eqs. (3) and (4) (with $b=a$ ) using the Hertz-Mindlin contact model (Table II). Then we fix $a_{0}(=a)$ or $\alpha_{0}$ in Eq. (5) by assuming $Z \approx 6$ and 4 for glass and PMMA bead packs, respectively; these are the values currently used in literature [20]. Figure 8 shows that the

TABLE II. Fit parameters from effective medium models.

\begin{tabular}{lcc}
\hline \hline Granular pack & $Z \alpha_{0}\left(P_{0}=1240 \mathrm{kPa}\right)$ & $Z \alpha_{0}\left(P_{0}=40 \mathrm{kPa}\right)$ \\
\hline glass beads & 0.154 & 0.048 \\
PMMA beads & 0.510 & 0.169 \\
\hline \hline
\end{tabular}

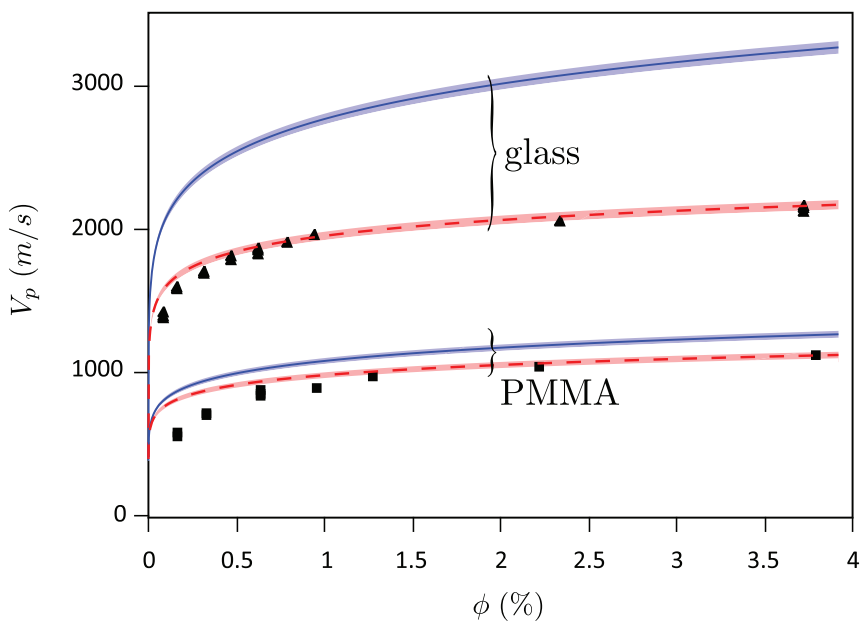

FIG. 8. (Color online) Comparisons of measured acoustic velocities $V_{P}$ in cemented glass and PMMA bead packs with those calculated by the bonded (solid blue lines) and cemented (dashed red lines) contact models, as a function of the cement content $\phi$.

bonded contact model agrees reasonably well with the data of cemented PMMA bead packs, but seriously deviates from those of glass bead packs.

\section{Cemented contact model}

Dvorkin et al. developed a bonded contact model for small amounts of cement at the bead contacts $(a \ll R)$, in which the elastic properties and content of the binder are considered [11]. For illustration, we show in Fig. 9 the radial distribution of normal stress $p(r)$ at a cemented contact $\left(\alpha_{0}=0\right)$ subject to a normal load $F_{n}$. In this elastic model, the surface of the cement is assumed to be infinitely rough and therefore no slip occurs at interfaces between the cement and grains. The thin cement layer is treated approximately as an elastic foundation with a normal displacement $\delta_{c}(r)=\left[p(r) / M_{c}\right] h(r)$ [24]. Here $M_{c}=2 G_{c}\left(1-v_{c}\right) /\left(1-2 v_{c}\right)$ is an elastic constant of the cement and $h(r)$ is its thickness [Fig. 9(a)]. Following the standard Hertz-Mindlin theory for the grain deformation [26], this model reveals a peculiar pattern of the normal stress $p(r)$ and also of the shear stress (not shown) at the cemented grain contacts as shown in Fig. 9(b): Both stresses are maximum at the center of the contact when the cement is soft compared to the grain and maximum at the periphery when the cement is stiff. Note that the adimensional parameter $\alpha / \Lambda_{n}$ adopted in this paper includes not only the ratio $\Lambda_{n}=M_{c}\left(1-v_{g}\right) / \pi G_{g}$ of the cement elastic constant $M_{c}$ to those of the grain, but also the amount of cement $\alpha=\alpha(\phi)$ [Eq. (5)]. We find that the soft cement and stiff cement regimes should correspond more precisely to $\alpha / \Lambda_{n} \gg 1$ and $\alpha / \Lambda_{n} \ll 1$, respectively.

Integrating the normal stress over the contact area yields the normal force $F_{n}$ in relation to the displacement $\delta_{n}=$ $\delta_{c}(r)+\delta_{g}(r)$, where $\delta_{g}$ is the surface displacement of the grain [Fig. 9(a)]. The normal stiffness computed from $D_{n}=$ $d F_{n} / d \delta_{n}$ can be expressed as $D_{n}=2 \pi R M_{c} k_{n}$, in which $k_{n}$ is calculated using a numerical method [11]. The tangential stiffness $D_{t}=d F_{t} / d \delta_{t}$ can also be evaluated using a similar approach in which $F_{t}$ and $\delta_{t}$ are the applied tangential force and displacement, respectively. As the cemented contact area 
(a)
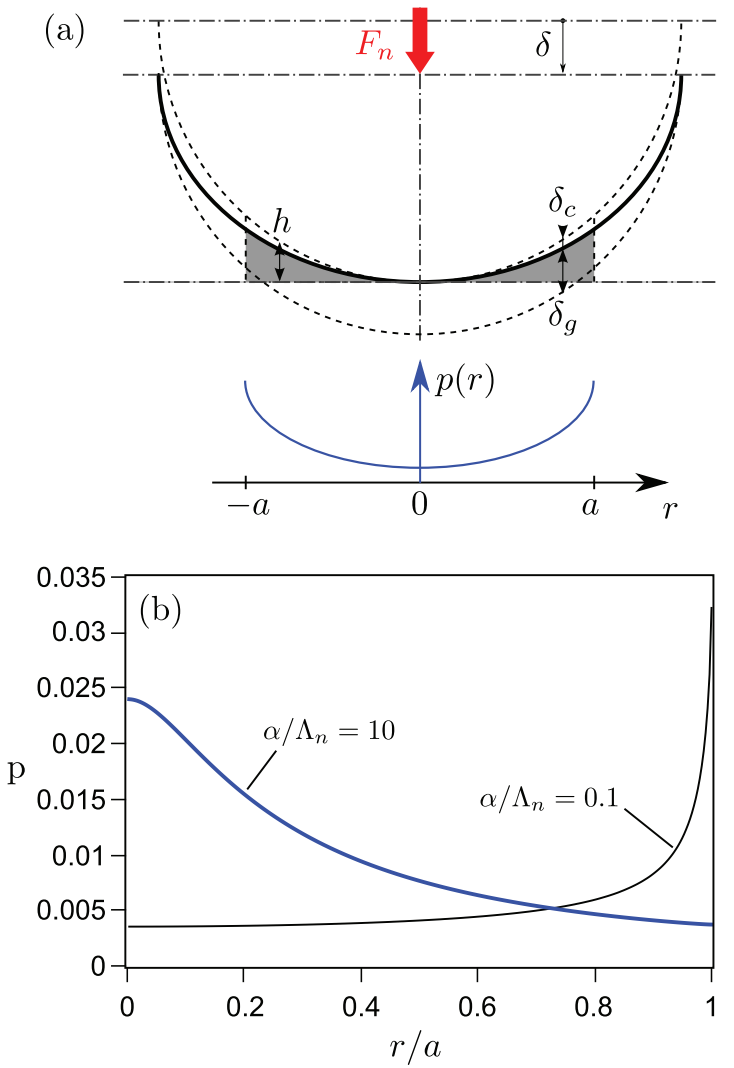

FIG. 9. (Color online) (a) Normal stress distribution at a cemented contact under a normal load $F_{n}$ for (b) two soft and stiff cements relative to the grains.

$\alpha$ is assumed constant in this model, the contact stiffnesses are independent of pressure.

We depict in Fig. 8 the sound velocities computed with these stiffnesses $D_{n}$ and $D_{t}$, using Eq. (1) and $Z a$ fitted with the data in the wet media as mentioned before. Compared to Digby's model, the prediction by the model of Dvorkin et al. agrees fairly well with the data in both cemented glass and PMMA bead packs. The discrepancy observed for the small amount of tetradecane concentration $\phi$ is likely due to the neglect of the liquid filling $\phi^{*}$ over the rough surface of the beads. Indeed, the best fit of $\phi^{*}$ via Eq. (5) with Figs. 4(a) and 4 (b) gives $\phi^{*}=0.17 \pm 0.03 \%$ for the glass beads and 0.06 $\pm 0.02 \%$ for PMMA beads. The values of the filling threshold are consistent with those in the previous works [2,3].

\section{Discussion}

The preceding comparisons with the measured acoustic velocities indicate that a pure interfacial bonding model (e.g., that of Digby) is not accurate enough to calculate the contact stiffness when the binder element is soft relative to the grain. The bulk elastic properties of the cementing materials should be taken into account in this case due to the significant deformation of the soft layer or cement bridge at the contact area $\left(\delta_{c}>\delta_{g}\right)$ under external load. As shown by a soft shell model [29], this situation may deviate seriously from the Hertz-like contact, where the geometrical nonlinearity plays a basic role due to the pressure dependence of the contact area between the grains.

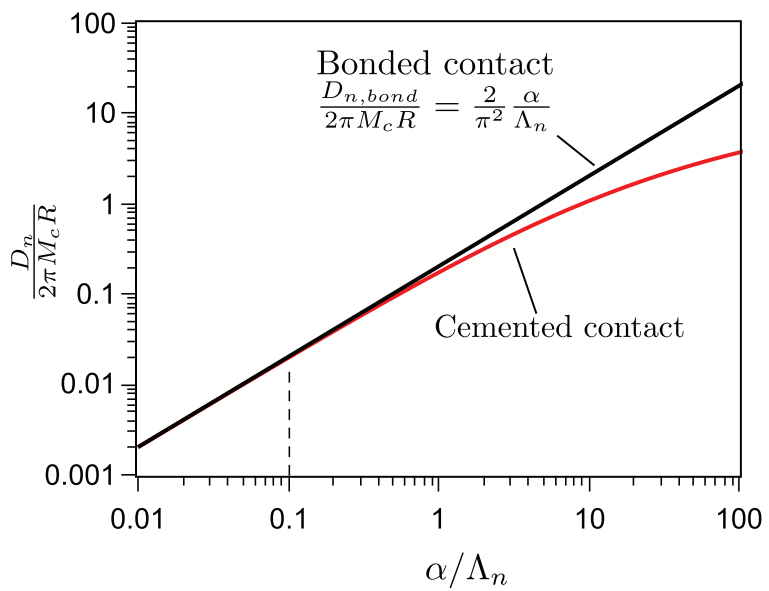

FIG. 10. (Color online) Comparison between the normal stiffnesses calculated from the bonded and cemented contact models, respectively.

We present in Fig. 10(a) a quantitative comparison between the normal contact stiffness $D_{n}$ predicted by the two bonded models used in this work. As shown by Eq. (3), the Digby model predicts a linear dependence of $D_{n}$ on the radius $a=$ $\alpha R$ of the bonded area, whereas the model of Dvorkin et al. predicts a smaller value of $D_{n}$, varying nonlinearly with $\alpha / \Lambda_{n}$. When $\alpha / \Lambda_{n}<0.1$ caused by either the small amount $(\alpha \leqslant 1)$ or the stiffness $\left(\Lambda_{n} \gg 1\right)$ of the cement relative to the grain, the two models predict almost the same contact stiffness with a difference less than $3 \%$. In this case, the properties of the cement material have little effect on the acoustic velocities in a cemented pack and Digby's model is a good approximation for describing a cemented contact.

Let us examine more precisely the prestress influence on the contact stiffness and the acoustic velocity. Two effects are noted: (i) the creation of a grain-grain contact by squeezing sideways the liquid (Fig. 1), enlarging the wetted (and cemented) contact area $\alpha$ at a given amount of cement, and (ii) the increase of the coordination or contact number $Z$. The first effect is local at the contact level [11], consequently enhancing the stiffness of the cemented contact $D$. Such an effect decreases when the cement content $\phi$ is increased [Fig. 4(c)]. The second issue involves the contact network at the mesoscopic scale in the wet granular packs. Indeed, it was shown that the confining pressure may increase $Z$ either by elastic compression of the grains or by buckling of the contact network [25]. The elastic modulus of the PMMA beads considered here is about ten times smaller than that of the glass beads. Moreover, the wet PMMA bead packs have a loose structure $\Phi \approx 0.59$ compared to those of glass bead packs $(\Phi \approx 0.62)$. These features would explain the stronger effect of prestress on the acoustic velocities observed in cemented PMMA bead packs shown in Fig. 4(c).

\section{ANALYSIS OF THE DAMAGE AT THE CEMENTED CONTACT}

A quantitative description of the irreversible behavior observed in Figs. 5 and 6 appears complicated. Here we seek to qualitatively interpret the experimental results with effective 
medium approaches, which take into account the elastic-plastic behavior of the cemented contact (Sec. IV A) and the prestress effect on the heterogeneity of the cemented granular material (Sec. IV B).

\section{A. Pressure sensitivity of the sound velocity under loading}

The pure elastic model for a cemented contact shown in Fig. 7(a) (deposition 1) predicts that the contact stiffness and consequently $V_{P}$ are independent of the applied pressure because of the constant area of a cemented contact [11]. For two elastic spheres uniformly coated with thin cemented layers (deposition 2), the contact stiffness may increase when the spheres are axially compressed together, due to the increasing contact area [12,29]. However, according to the amount of added liquid, the deposition of the cement in this work likely corresponds to deposition 3, quite different from deposition 2 .

To understand the pressure dependence of the acoustic velocity $V_{P}$ [Figs. 5(a), 5(c), and 6], we investigate the effect of the plasticity in cemented granular materials. For the dense packs of cemented glass and PMMA beads considered here, the plasticity induced by the large strain is mostly due to the plastic nature of the cemented contacts via the contact stiffness rather than the structure change such as the packing density $\Phi$ or mean coordination number $Z$ [Eq. (3)]. Dvorkin et al. showed that [13] if the cement is soft compared to the grains, a plastic zone may develop around the center of the contact (where the stress is maximum) as the external load $F$ increases. As the cement is squeezed sideways, a (Hertz-like) finite direct contact area may develop between the grains, resulting in larger contact stiffness at a given confining pressure. This scenario is consistent with our data in glass bead packs [Fig. 6(a)].

For cemented PMMA bead packs, the cement is stiffer than (or similar to) the grains. As a result, the maximum stress is localized on the periphery of the contact area where the plastic flow is expected to begin. Instead, to develop the direct grain-grain contact area as done with cemented glass beads, we would expect a significant increase of the cemented contact area $\alpha=a / R$, due to the plastic flow or a fracture process initiated at the grain-cement interface. This mechanism may also enhance the contact stiffness. For very small amounts of cement $\phi<0.5 \%$, the shear strength of the cemented contacts should be weaker. Furthermore, the relative increase in the contact area $\alpha$ via the plastic flow would be more significant at small values of $\phi$ due to geometric considerations. Such a picture would explain the high-pressure sensitivity of the acoustic velocity observed with small $\phi$ [Fig. 6(b)].

\section{B. Damage of the cemented contact under unloading}

The cemented bead pack prepared with high prestress $P=$ $1250 \mathrm{kPa}$ undergoes the important decrease in sound velocity as the confining pressure is progressively removed [Fig. 5(b)]. The resulting tensile would cause partly the debonding or fracture at the cement-grain interface or inside the cement, at the center or periphery of the cemented contact where the stress is maximum (Fig. 11). This may decrease the size of the contact area and consequently reduces the contact stiffness and the acoustic velocity. Moreover, the observation in Fig. 5(d) with the correlation technique of the coda waves suggests

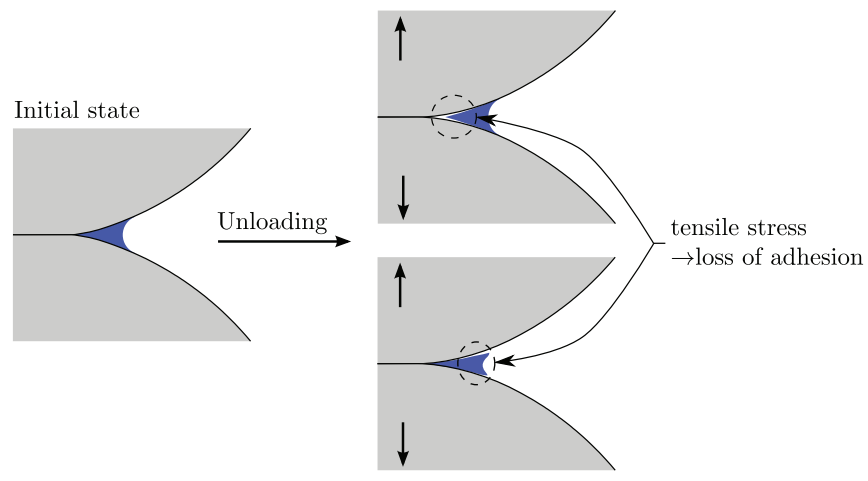

FIG. 11. (Color online) Possible fractures at the cemented contact, prepared with high stress, upon unloading.

that the damage caused by the unloading is important, likely transforming the material into a heterogeneously fractured medium. This would be caused by the highly inhomogeneous stress field in the wet glass bead pack under applied high (pre)stress; such residual heterogeneity of stress remains inside the cemented pack and results in a heterogeneous tensile when unloading. Under reloading, the fractured medium could be partly healed and the acoustic velocity may increase as in noncohesive granular packs.

For the packs of bead cemented under low prestress $P_{1}$ and loaded to a higher stress $P_{2}$ unloading might also cause the development of cracks in the plastic zones at the cement-grain interfaces, created during the first loading. Under unloading or reloading, Fig. 5(b) suggests that the damage in such cemented material progressively develops or heals, probably due to a quite homogeneous distribution of the cracks at the cement-grain interfaces and the absence of strongly heterogeneous residual stresses (Fig. 11). Further work is needed to understand the interplay between the interfacial adhesion and fracture and the plastic behavior of the cement at the contact level during loading and unloading.

\section{CONCLUSION}

Measurements of the acoustic velocity of compressional waves have been performed during the controlled cementation of glass and PMMA bead packs wet by tetradecane. Our results show that the effective elastic moduli of weakly cemented dense granular packs are basically determined by the contact stiffnesses between the beads. The quantitative agreement between the elastic model of the cemented contact and the data confirms the importance of the bulk mechanical property of the cementing material, a kind of third body between the grains. A bonded contact model provides a good approximation for either the stiff cement relative to the grain or a small amount of cement, but fails to quantitatively explain the data when the cement is soft.

The pressure dependence of the acoustic velocity in these cemented samples is mostly associated with the adhesive elastic-plastic nature of the cemented contact between the grains. In glass bead packs, external loading would develop a stiffer grain-grain contact, whereas in PMMA bead packs it may increase the size of the cemented contact. By unloading cemented granular samples prepared at high prestress, acoustic 


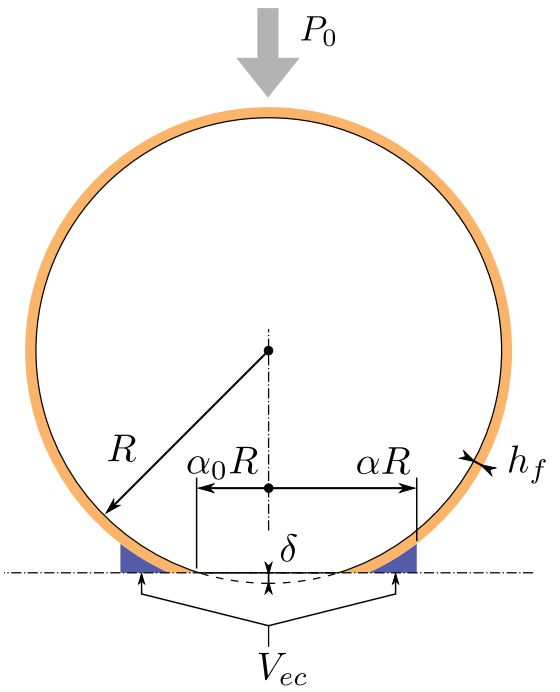

FIG. 12. (Color online) Deposition of the wetting liquid or cement around a rough sphere.

velocities decrease. The correlation technique of the codalike scattered waves shows that this irreversible behavior is likely associated with the heterogeneously fractured structure caused by tensile stress, due to the strongly inhomogeneous residual stress. On the contrary, the cemented material prepared at low prestress develops a more progressive damage process under unloading, likely due to the cracks at the cement-grain interfaces distributed more evenly inside the cemented contact network. More investigation is necessary to understand the respective contributions from the interfacial adhesion and fracture and the plastic behavior or breakage of the cementing materials.
This work shows that acoustic measurements allow probing the complex mechanical properties on scales of the contact in cemented granular materials. We believe that further acoustic absorption measurements via diffusing wave techniques [7] would provide more information on these dissipative contacts.

\section{APPENDIX: DISTRIBUTION OF THE WETTING LIQUID AROUND A ROUGH SPHERE}

Let us consider the distribution of the liquid around a sphere of radius $R$ in disposition 3 [Fig. 7(a)]. In the presence of the prestress $P_{0}$, we assume that the coating layer is squeezed out of the contact zone between the spheres as shown in Fig. 12. The relative approach $\delta$ of the spheres under $P_{0}$ is related to the radius $a_{0}$ of the contact area by $a_{0}^{2} \approx 2 \delta R$. The volume of the pendular ring (or liquid bridge) depends on the normalized radius $\alpha, \alpha_{0}$, and the thickness of the coating film $\bar{h}_{f}\left(=h_{f} / R\right): V_{\text {ring }} \approx(3 / 16)\left(\alpha^{2}-\alpha_{0}^{2}-2 \bar{h}_{f}\right)^{2} V_{\text {sphere }}$.

For a small amount of liquid (or cement) $\phi<\phi^{*}$, the wetting liquid would distribute around the sphere as a coating film with thickness $h_{f}=\phi R / 3$. Under $P_{0}$, the coating film of volume $V_{\text {film }} \approx(3 / 2)(\delta / R)\left(h_{f} / R\right) V_{\text {sphere }}=(\delta \phi / 2 R) V_{\text {sphere }}$ is squeezed sideways to the periphery of the contact. Setting $V_{\text {ring }}=V_{\text {film }}$ yields

$$
\alpha=\left[\alpha_{0}^{2}+2 \phi / 3+\left(4 \alpha_{0}^{2} \phi / 3\right)^{1 / 2}\right]^{1 / 2} .
$$

For a volume of the wetting liquid $\phi>\phi^{*}$, the thickness of the coating film almost remains unchanged at $h_{f}=\phi^{*} R / 3$ and the excess of the volume $\left(\phi-\phi^{*}\right) V_{\text {sphere }} / Z$ migrates to the contact zone between the spheres. Adding to the squeezed liquid $V_{\text {film }}^{*} \approx\left(\delta \phi^{*} / 2 R\right) V_{\text {sphere }}$, one obtains the radius $\alpha$ of the pendular ring,

$$
\alpha=\left\{2 \phi^{*} / 3+\alpha_{0}^{2}+\left[4 \alpha_{0}^{2} \phi^{*} / 3+16\left(\phi-\phi^{*}\right) / 3 Z\right]^{1 / 2}\right\}^{1 / 2} .
$$

[1] L. Bocquet, E. Charlaix, S. Ciliberto, and J. Crassous, Nature (London) 396, 735 (1998); N. Fraysse, H. Thomé, and L. Petit, Eur. Phys. J. B 11, 615 (1999).

[2] P. Tegzes, R. Albert, M. Paskvan, A.-L. Barabási, T. Vicsek, and P. Schiffer, Phys. Rev. E 60, 5823 (1999).

[3] T. G. Mason, A. J. Levine, D. Ertas, and T. C. Halsey, Phys. Rev. E 60, R5044 (1999); T. C. Halsey and A. J. Levine, Phys. Rev. Lett. 80, 3141 (1998).

[4] S. Nowak, A. Samadani, and A. Kudrolli, Nat. Phys. 1, 50 (2005); S. Herminghaus, Adv. Phys. 54, 221 (2005); N. Mitarai and F. Nori, ibid. 55, 1 (2006).

[5] C. C. Pilbeam and J. R. Vaisneys, J. Geophys. Res. 78, 810 (1973).

[6] J. J. Valenza, C.-J. Hsu, R. A. Ingale, N. Gland, H. A. Makse, and D. L. Johnson, Phys. Rev. E 80, 051304 (2009).

[7] X. Jia, Phys. Rev. Lett. 93, 154303 (2004); T. Brunet, X. Jia, and P. Mills, ibid. 101, 138001 (2008).

[8] X. Jia and P. Mills, in Powders and Grains, edited by Y. Kishino (Balkema, Rotterdam, 2001)

[9] P. J. Digby, J. Appl. Mech. 48, 803 (1981).

[10] K. W. Winkler, Geophys. Res. Lett. 10, 1073 (1983).
[11] J. Dvorkin, A. Nur, and H. Yin, Mech. Mater. 18, 351 (1994); J. Dvorkin and H. Yin, Int. J. Solid Struct. 32, 2497 (1995);

J. Dvorkin and A. Nur, Geophysics 61, 1363 (1996).

[12] D. Elata and J. Dvorkin, Mech. Mater. 23, 147 (1996).

[13] J. Dvorkin and D. Yale, Comput. Geotech. 20, 287 (1997); J. Dvorkin, Mech. Mater. 23, 29 (1996).

[14] V. Topin, J.-Y. Delenne, F. Radjai, L. Brendel, and F. Mabille, Eur. Phys. J. E 23, 413 (2007); S. Luding, K. Manetsberger, and J. Mullers, J. Mech. Phys. Solids 53, 455 (2005).

[15] A. Castellanos, Adv. Phys. 54, 263 (2005).

[16] R. Snieder and J. Page, Phys. Today 60(4), 49 (2007); N. Tremblay, E. Larose, and V. Rossetto, J. Acoust. Soc. Am. 127, 1239 (2010).

[17] X. Jia, J. Laurent, Y. Khidas, and V. Langlois, Chin. Sci. Bull. 54, 4327 (2009).

[18] X. Jia, C. Caroli, and B. Velicky, Phys. Rev. Lett. 82, 1863 (1999); B. Gilles and C. Coste, ibid. 90, 174302 (2003).

[19] B. Audoin and J. Roux, Ultrasonics 34, 25 (1996).

[20] H. A. Makse, N. Gland, D. L. Johnson, and L. Schwartz, Phys. Rev. E 70, 061302 (2004); J. Jenkins, D. Johnson, L. La Ragione, and H. Makse, J. Mech. Phys. Solid 53, 197 (2005). 
[21] I. Yu. Solodov, Ultrasonics 36, 383 (1998); L. A. Ostrovsky and P. A. Johnson, Rivista del Nuovo Cimento 24, 1 (2001); K. Van Den Abeele, P. Y. Le Bas, B. Van Damme, and T. Katkowski, J. Acoust. Soc. Am. 126, 963 (2009); N. Chigarev, J. Zakrzewski, V. Tournat, and V. Gusev, J. Appl. Phys. 106, 036101 (2009).

[22] V. F. Nesterenko, Dynamics of Heterogeneous Materials (Springer, New York, 2001); C. Daraio, V. F. Nesterenko, E. B. Herbold, and S. Jin, Phys. Rev. Lett. 96, 058002 (2006); S. Sen et al., Phys. Rep. 462, 21 (2008); E. B. Herbold and V. F. Nesterenko, Phys. Rev. E 75, 021304 (2007); V. Tournat, V. Zaitsev, V. Nazarov, V. Gusev, and B. Castagnède, Acoust. Phys. 51, 543 (2005); T. Brunet, X. Jia, and P. Johnson, Geophys. Res. Lett. 35, L19308 (2008).
[23] C.-h. Liu and S. R. Nagel, Phys. Rev. Lett. 68, 2301 (1992); S. R. Hostler and C. E. Brennen, Phys. Rev. E 72, 031303 (2005); E. T. Owens and K. Daniels, Europhys. Lett. 94, 54005 (2011).

[24] S. Wildenberg, M. van Hecke, and X. Jia, Europhys. Lett. 101, 14004 (2013); X. Jia, T. Brunet, and J. Laurent, Phys. Rev. E 84, 020301(R) (2011).

[25] J. D. Goddard, Proc. R. Soc. London Ser. A 430, 105 (1990).

[26] K. L. Johnson, Contact Mechanics (Cambridge University Press, Cambridge, 1985).

[27] A. N. Norris and D. L. Johnson, J. Appl. Mech. 64, 39 (1997).

[28] V. Langlois and X. Jia, Powder Technol. 208, 509 (2011).

[29] P.-G. de Gennes, Europhys. Lett. 35, 145 (1996). 